\title{
Use of data mining to determine changes in the gene expression profiles of rat embryos following prenatal exposure to inflammatory stimulants
}

\author{
JIANZHI ZHOU，XINGXING ZHANG，HAIGANG ZHANG，YI JIA，YA LIU，YUAN TANG and XIAOHUI LI \\ Institute of Materia Medical, College of Pharmacy, Third Military Medical University, Chongqing 400038, P.R. China
}

Received November 22, 2012; Accepted May 16, 2013

DOI: $10.3892 / \mathrm{mmr} .2013 .1498$

\begin{abstract}
Numerous clinical epidemiology and laboratory studies have demonstrated a close association between inflammation or immunity and the occurrence of hypertension. Our group has previously shown that upon intraperitoneal injection of lipopolysaccharide (LPS) or zymosan (Zym) into pregnant rats, the offspring of the experimental group exhibited higher blood pressure levels compared with the control group, and these may be associated with the effects of an accumulation of gene expression changes on female rats during pregnancy. The present study used the Affymetrix GeneChip ${ }^{\circledR}$ Rat Genome 2302.0 Array to examine gene expression profile changes in the embryos of experimental pregnant rats intraperitoneally injected with LPS or Zym vs. the untreated controls. We discovered that genes associated with DNA replication and mRNA processing were significantly upregulated by the stimuli from analysis using GenMAPP. By contrast, genes involved in the calcium regulatory pathway in cardiac myocytes and the signaling pathway in smooth muscle contraction and relaxation were the most downregulated. Results of the microarray analysis showed that immuno-inflammatory stimuli during pregnancy affected the gene expression profiles of the embryos. These changes in gene expression may affect the developmental and metabolic status of the offspring, thereby increasing their susceptibility to hypertension and obesity.
\end{abstract}

\section{Introduction}

The 'fetal origins of adult chronic diseases' theory has been proposed in modern developmental biology. This theory suggests that several adult chronic diseases, including coronary heart disease, type II diabetes and hypertension, originate during developmental plasticity in the embryonic and infant

Correspondence to: Professor Xiaohui Li, Institute of Materia Medical, College of Pharmacy, Third Military Medical University, 30 Gaotanyan Main St, Chongqing 400038, P.R. China

E-mail:1psh008@aliyun.com

Key words: inflammation, embryo, gene expression profile, lipopolysaccharide, zymosan, signaling process stages (1-3). Therefore, external stimuli during pregnancy may affect embryonic development and play a key role in adult chronic diseases (4). One of these diseases is essential hypertension (EH), which is a common cardiovascular illness. Although its etiology has not been fully elucidated, it has been shown that the occurrence of EH is associated with the interaction between congenital genetic susceptibility and acquired environmental factors. Previous studies have mainly focused on the identification of relevant susceptible genes, including the use of candidate gene approaches and genome-wide scanning (4). To date, $>100$ genes have been studied, including those involved in the reninangiotensin-aldosterone system, adrenergic receptors of the sympathetic nervous system, endothelial functional proteins, G-protein signaling proteins, cytokines and ion channels (5-7). Although a number of these genes have been shown to be associated with EH, they do not fully explain its occurrence.

Previous studies have shown that inflammation is closely associated withEH (8,9). In 2002, Parissis et al (10)demonstrated that the expression of inflammatory cytokines in the peripheral blood of EH patients was increased, and that the increased inflammatory cytokine levels during hypertension were mainly related to peripheral vascular inflammation and endothelial cell dysfunction. Studies by Samuelsson et al $(11,12)$ reported that following the stimulation of pregnant rats with IL-6, the offspring exhibited elevated blood pressure levels, obesity and enhanced activity of the hypothalamic-pituitary-adrenal axis. Since hypertension was caused by one single cytokine in these studies, this suggests that inflammation may be one of the direct causes of EH. However, although the level of inflammatory cytokine IL-6 is significantly increased during inflammation, IL-6 levels alone do not reflect the immune-inflammatory status of the body (13).

Based on the available literature and our experimental studies, we hypothesized that prenatal maternal inflammation is important in EH pathogenesis. In previous studies conducted by our group, lipopolysaccharide (LPS) from the cell wall of Gram-negative bacteria and zymosan (Zym), both capable of initiating systemic and non-specific inflammatory immune responses, were used as immuno-inflammatory stimuli. Either LPS or Zym was intraperitoneally injected into female rats on gestational days 8, 10 and 12. The 6-week-old offspring of these rats displayed significantly higher blood pressure levels compared with the respective controls, and at 24 weeks, a hyper- 

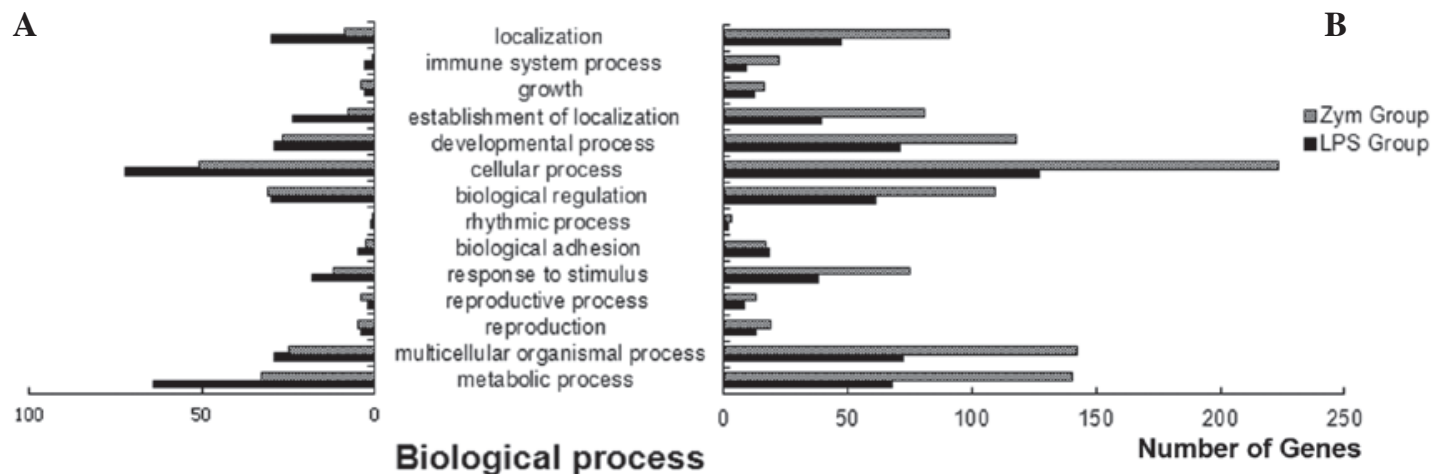

Figure 1. Expression profiles of genes involved in various biological processes. (A) Upregulated and (B) downregulated genes. The Zym and LPS groups were compared with the control group using the GeneChip Rat Genome 2302.0 Array. Gray bars indicate prenatal exposure to lipopolysaccharide (LPS); black bars indicate prenatal exposure to zymosan (Zym).

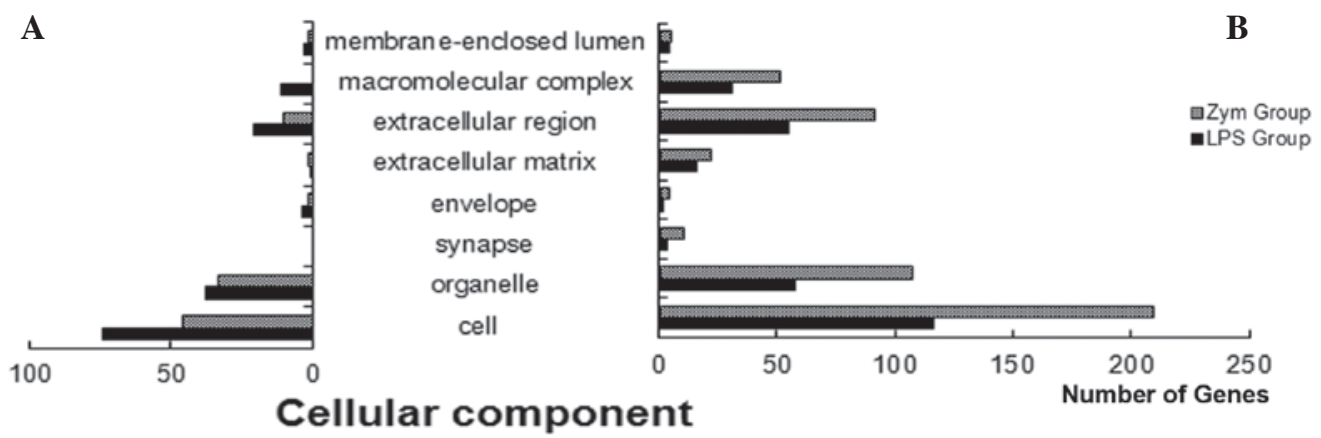

Figure 2. Expression profiles of genes involved in various cellular components. (A) Upregulated and (B) downregulated genes. The Zym and LPS groups were compared with the control group using the GeneChip Rat Genome 2302.0 Array. Gray bars indicate prenatal exposure to lipopolysaccharide (LPS); black bars indicate prenatal exposure to zymosan (Zym).

A

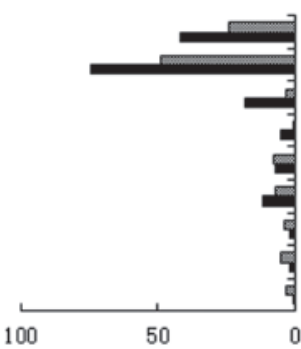

catalytic activity binding transporter activity transcription regulator activity structural molecule activity signal transducer activity motor activity enzyme regulator activity antioxidant activity

\section{Molecular function}

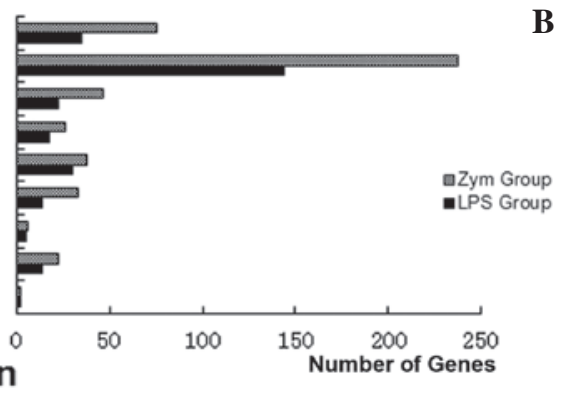

B

Figure 3. Expression profiles of genes involved in various molecular functions. (A) Upregulated and (B) downregulated genes. The Zym and LPS groups were compared with the control group using the GeneChip Rat Genome 230 2.0 Array. Gray bars indicate prenatal exposure to lipopolysaccharide (LPS); black bars indicate prenatal exposure to zymosan (Zym).

tension level of $140 \mathrm{mmHg}$ was observed. Furthermore, the body weights of offspring in the experimental group were significantly increased compared with that of the controls (14-17).

In the present study, the Affymetrix GeneChip ${ }^{\circledast}$ Rat Genome 2302.0 Array was used to investigate expression profile changes in the embryos after pregnant female rats were intraperitoneally injected with LPS or Zym. Additionally, fluorescent quantitative (q)PCR was used to validate the differentially expressed genes determined using the microarray analysis. The results of this study shed light on embryonic development following immuneinflammatory stimulation during pregnancy, providing valuable information for future studies investigating the association between hypertension and inflammation.

\section{Materials and methods}

Animals. Pregnant Sprague-Dawley rats (200-250 g) were purchased from the Experimental Animal Center of the Third Military Medical University (Chongqing, China). The rats were raised under the following conditions: $23-25^{\circ} \mathrm{C}, 60 \%$ relative humidity, 12-h light/dark cycle, periodic air changes (every $15 \mathrm{~min}$ ) and ad libitum access to food and water. This study was performed in strict accordance with recommendations in the GuidefortheCare andUse ofLaboratory Animals published by the National Institutes of Health (NIH Publication no. 85-23, revised 1996; http://www.nap.edu/openbook.php?record_id=5140). The protocol was approved by the Ethics Committee for Animal 
A

DNA replication

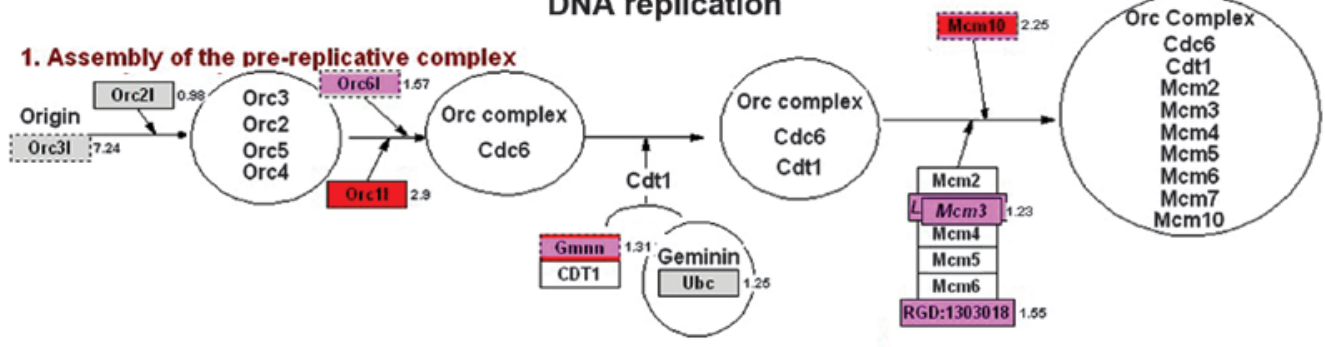

2. Activation of the pre-replicative complex

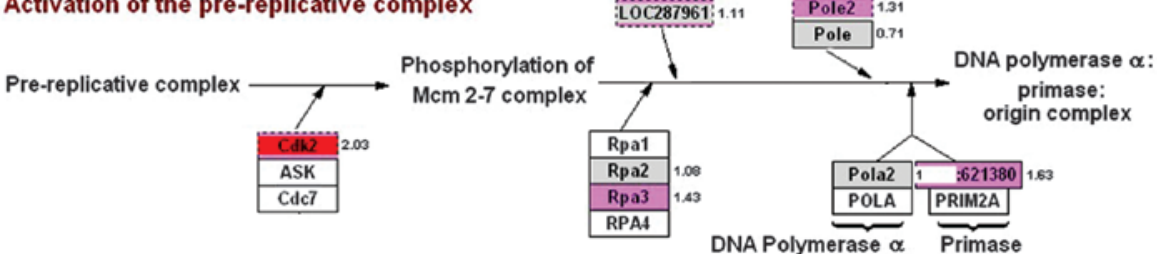

3. Leading/lagging strand synthesis

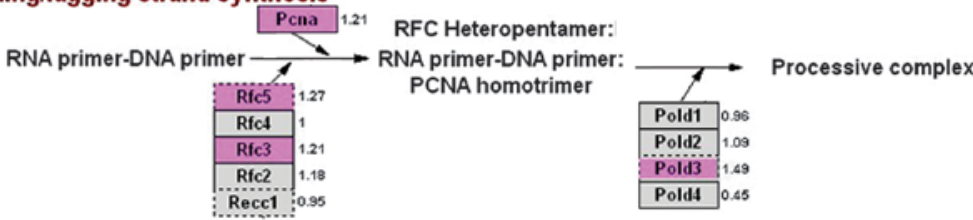

B

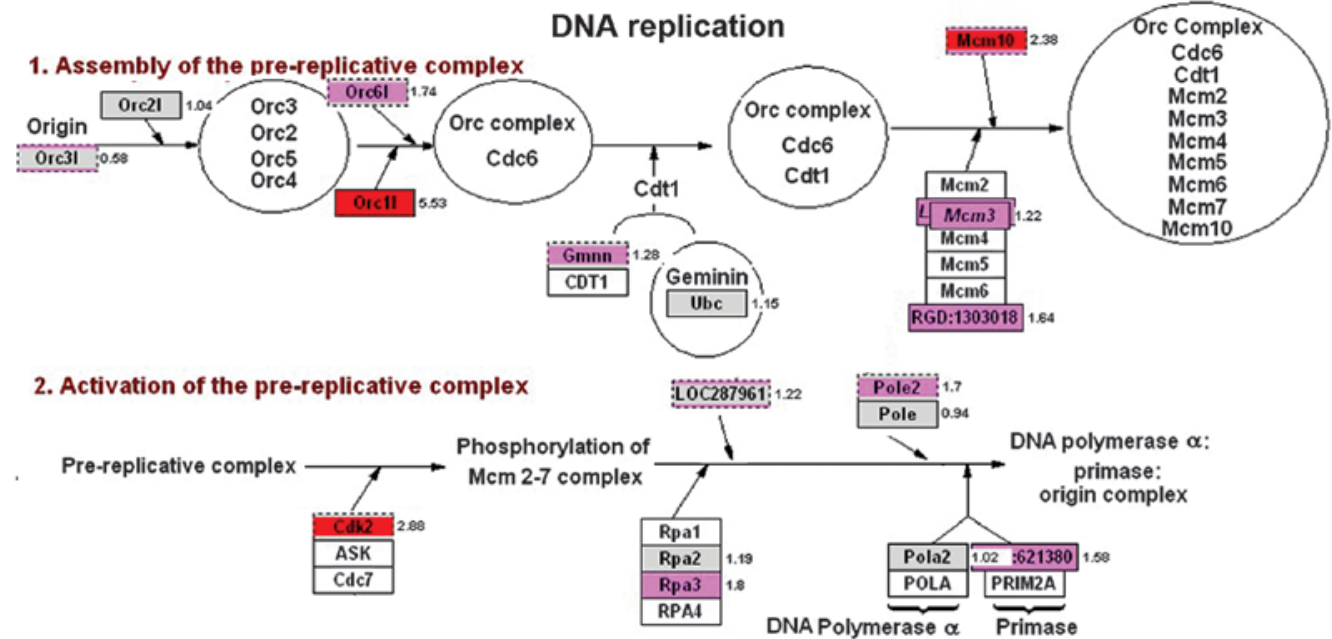

3. Leading/lagging strand synthesis

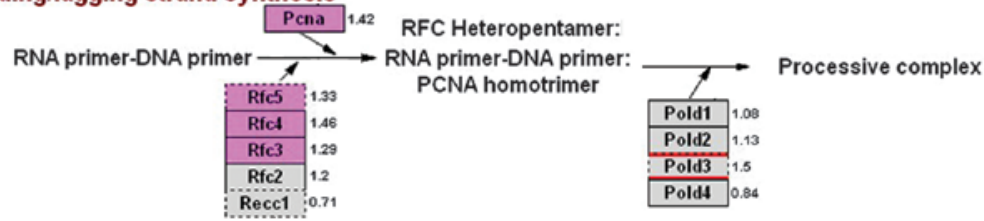

Figure 4. Analysis of signaling processes involved in DNA replication. Gene expression profiles of rat embryos prenatally exposed to (A) lipopolysaccharide (LPS) and (B) zymosan (Zym). The signaling process diagrams were overlaid with gene-expression color criteria and ratios of gene-expression using the GenMAPP program. Red, upregulated (ratio >2); pink, upregulated (ratio >1.2); grey, no significant change; white, gene not expressed on the GeneChip Array.

Experimentation of the Third Military Medical University. All the surgical procedures were performed under ether anesthesia and all efforts were made to minimize suffering.

Model preparation. Fifteen pregnant rats were randomly divided into three groups (5 animals/group); the control, LPS and Zym groups and animals in these groups were intraperitoneally injected with $1 \mathrm{ml} / \mathrm{kg}$ sterile saline, $0.79 \mathrm{mg} / \mathrm{kg}$ LPS or $8 \mathrm{mg} / \mathrm{kg}$ Zym, respectively. The solutions were administered between 8:00 and 9:00 a.m. on gestational days 8, 10 and 12 . Twelve hours after the final injection, the rats were anesthe- tized and caesarean sections were performed immediately under sterile conditions in order to obtain the embryos. One embryo was removed from each pregnant rat and placed into an RNAlater solution, which was incubated at $4^{\circ} \mathrm{C}$ overnight and then stored at $-80^{\circ} \mathrm{C}$ (Qiagen, Mainz, Germany).

Extraction of total RNA. Each whole embryo was homogenized and the total RNA was extracted using the Qiagen RNA extraction kit (Qiagen). Total RNA then was purified using the Qiagen RNeasy Mini Kit (total RNA yield, $>45 \mu \mathrm{g}$; Qiagen). 
A

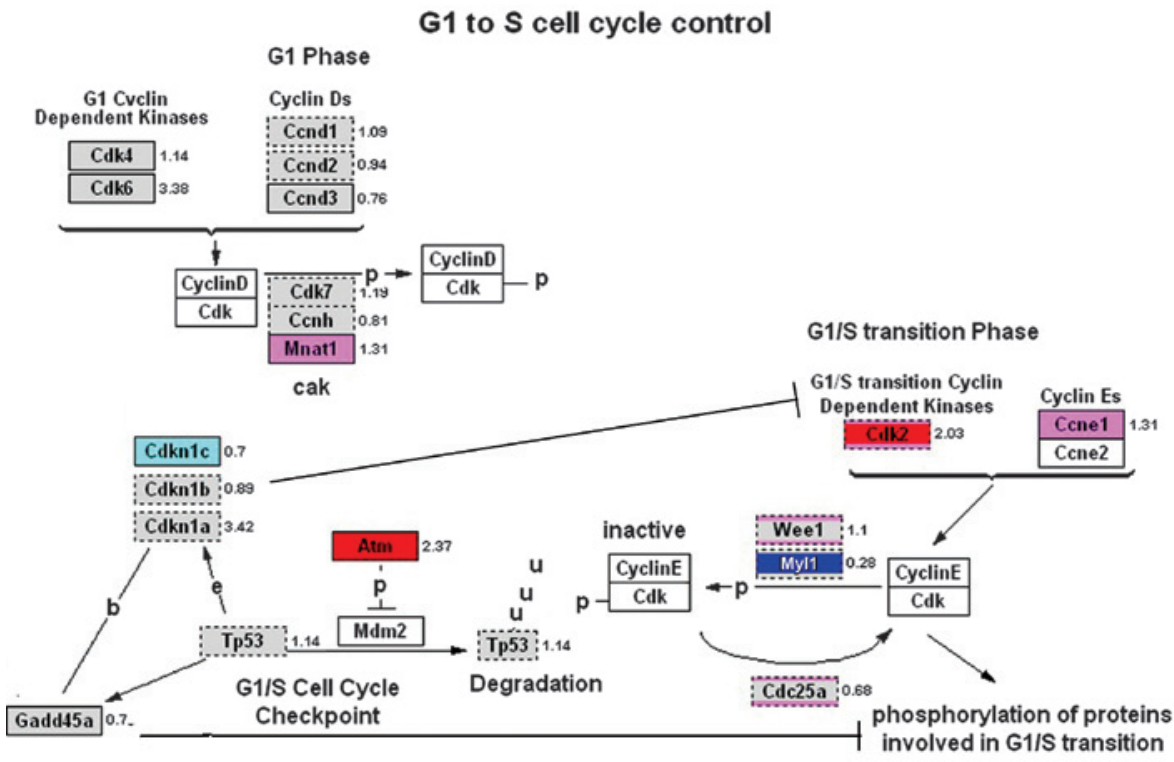

$\mathbf{B}$

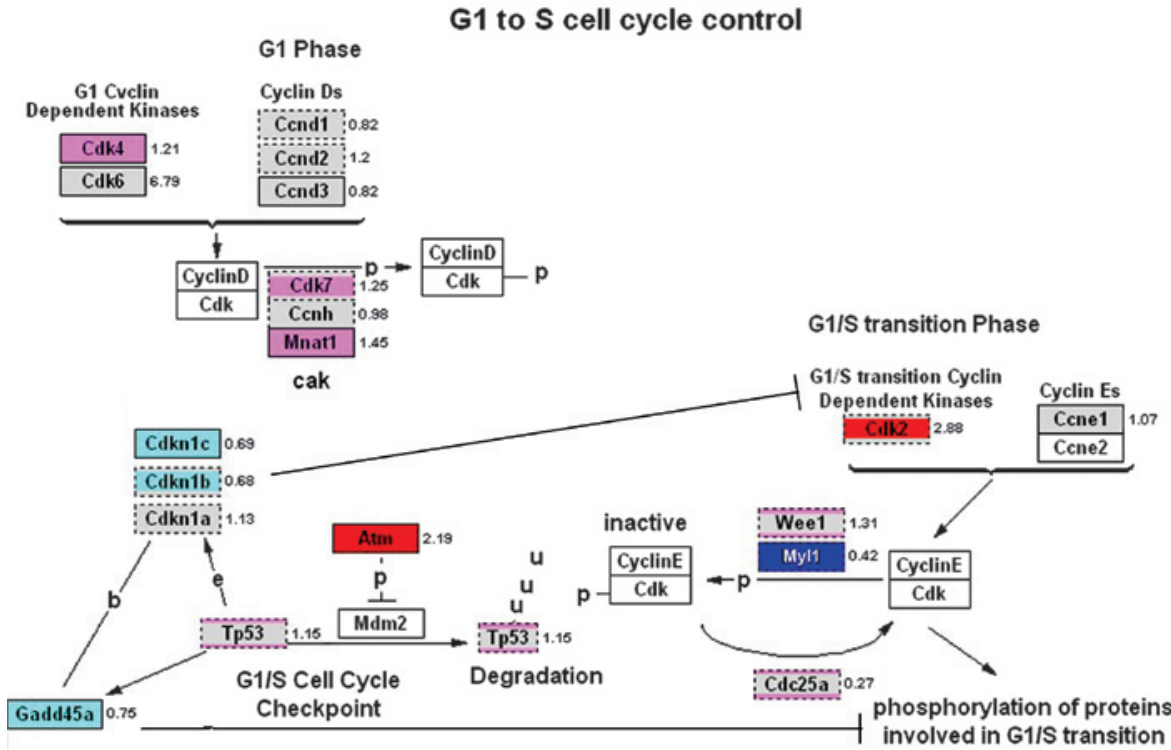

Figure 5. Analysis of signaling processes that regulate the G1 to S phase transition of the cell cycle. Gene expression profiles of rat embryos prenatally exposed to (A) lipopolysaccharide (LPS) and (B) zymosan (Zym). The signaling process diagrams were overlaid with gene-expression color criteria and ratios of gene-expression using the GenMAPP program. Red, upregulated (ratio >2); pink, upregulated (ratio >1.2); blue, downregulated (ratio >2); light blue, downregulated (ratio >1.2); grey, no significant change; white, gene not expressed on the GeneChip Array.

Microarray hybridization. The One-Cycle cDNA Synthesis kit (Affymetrix Inc., Santa Clara, CA, USA) was used to generate first- and second-strand cDNA from a sample of total RNA $(1-8 \mu \mathrm{g})$. The synthesized cDNA was purified using the cDNA Cleanup Spin Column (Affymetrix Inc.). The cRNA was then synthesized and purified using the GeneChip IVT Labeling kit, then fragmented to a size range of 35-200 bp for microarray hybridization. The microarray hybridization was performed by Gene Tech Co., Ltd. (Shanghai, China). The differentially expressed genes were determined based on the following two criteria: i) signal ratios of $>2.0$ and $<0.5$ were considered to be upregulated and downregulated genes, respectively; and ii) at least one signal of the two signal detection values was required to be significantly higher than the backgroud value.

Data analysis. Gene expression changes following LPS and Zym stimulation were subjected to pathway analysis using the GenMAPP and MAPPFinder programs (http://www.genmapp.org). Functional analysis was performed based on the biological function classification in the Gene Ontology (GO) database (http://www.geneontology.org). More specifically, the analysis was performed using three major characteristics; biological processes, molecular functions and cellular components. GenMAPP classifies signaling pathways into four major groups; cellular processes, metabolic processes, molecular functions and physiological processes. All the expression profile data from the LPS vs. control and Zym vs. control experiments were first compiled using the document format required by GenMAPP. Subsequently, the two data sets were imported into the Expression Dataset Manager of the GenMAPP software. In the color sets, the colors were used to indicate the up- and downregulated genes, as well as the fold-changes for gene expression. 
mRNA processing

Processing of Intron-Containing Pre-mRNA

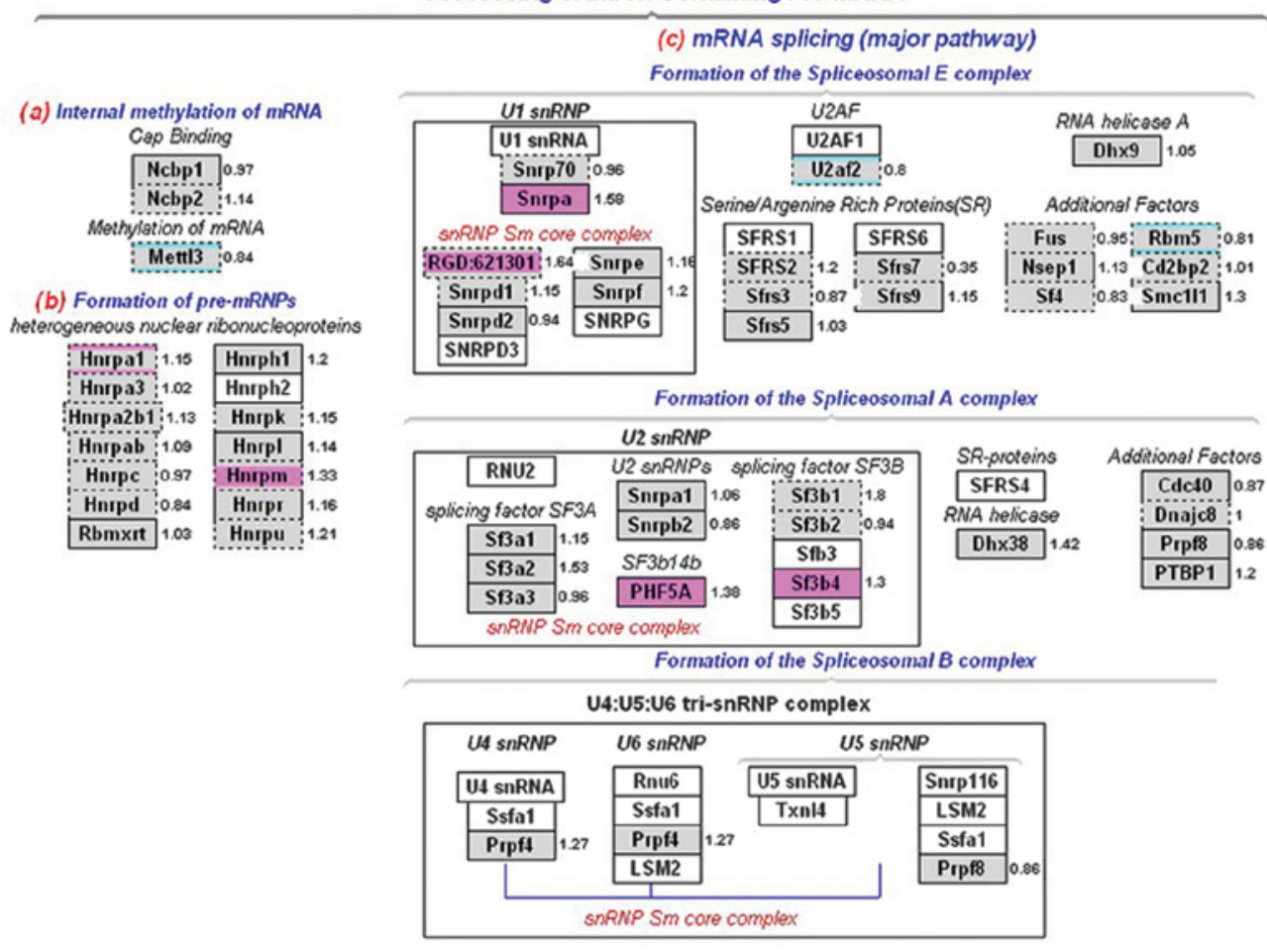

$\mathbf{B}$

mRNA processing

Processing of Intron-Containing Pre-mRNA

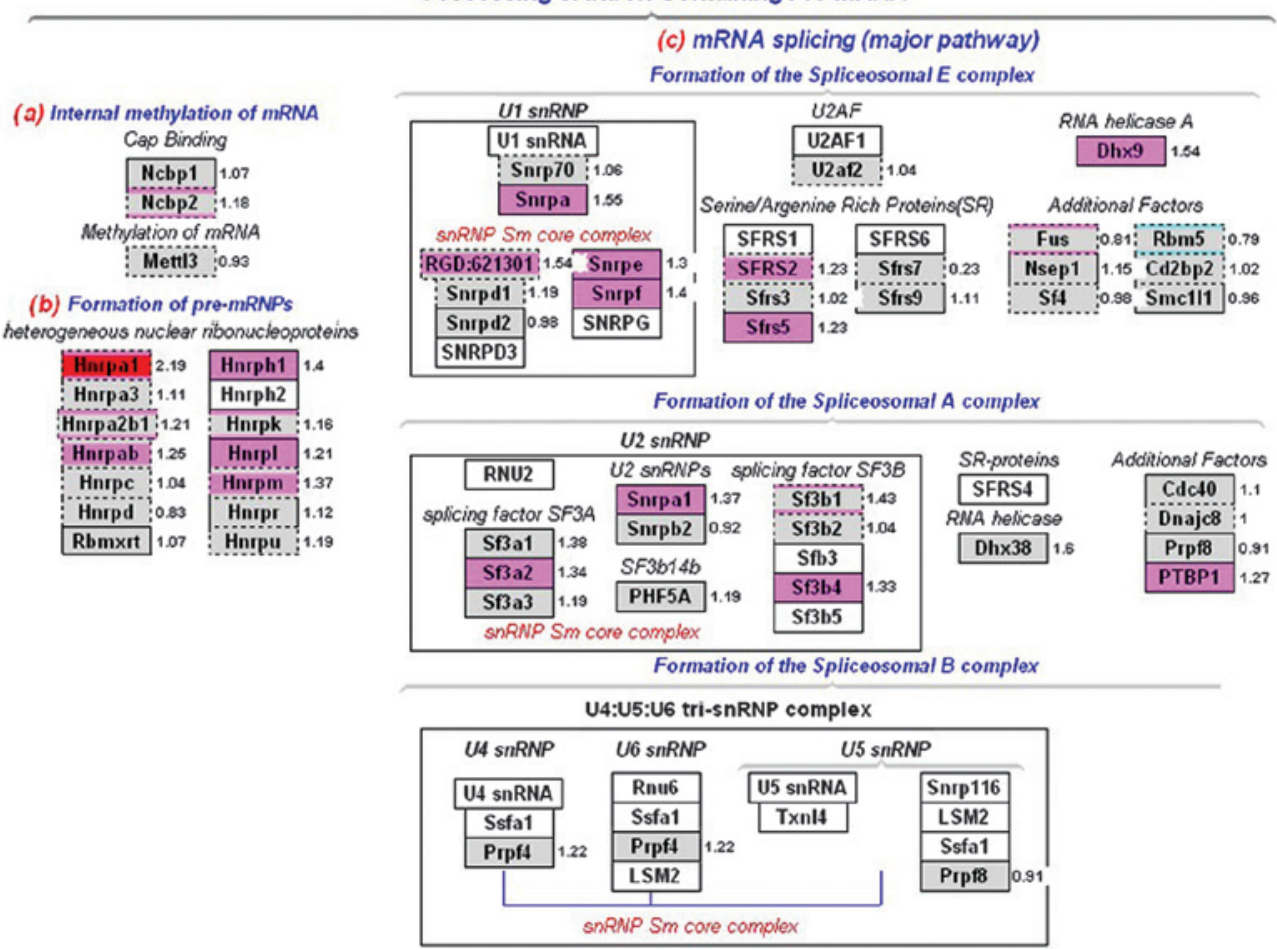

Figure 6. Analysis of signaling processes involved in mRNA processing. Gene expression profiles of rat embryos prenatally exposed to (A) lipopolysaccharide (LPS) and (B) zymosan (Zym). The signaling process diagrams were overlaid with gene-expression color criteria and ratios of gene-expression using the GenMAPP program. Red, upregulated (ratio $>2$ ); pink, upregulated (ratio >1.2); light blue, downregulated (ratio >1.2); grey, no significant change; white, gene not expressed on the GeneChip Array.

$q P C R$. Twenty genes of interest were selected to be examined using qPCR. Total RNA extraction and cDNA generation were performed according to the manufacturer's instructions
(Takara Biomedical Technology, Beijing, China). Detection was performed using the $\mathrm{SYBR}^{\circledR}$ Green PCR Master mix (Takara Biomedical Technology). The double-standard curve 
A
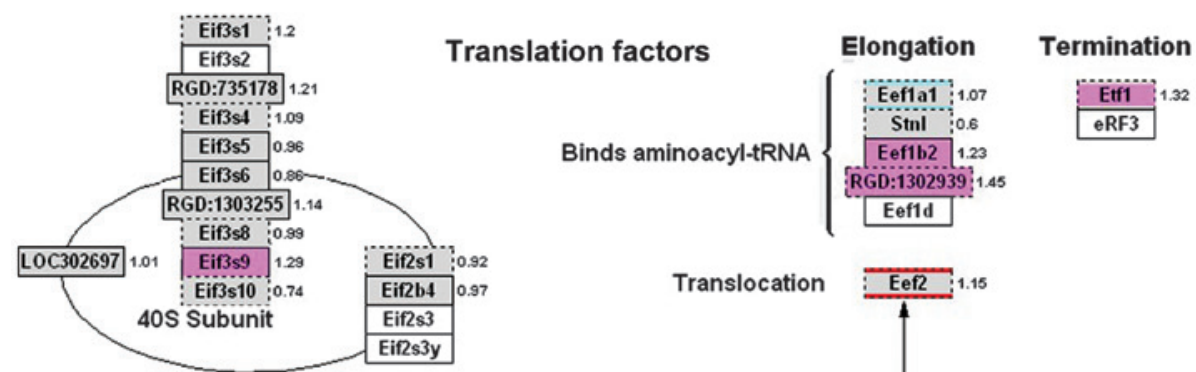

Translocation

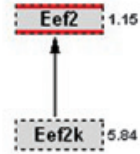

43S Pre-initiation Complex

Eef2k 5.84

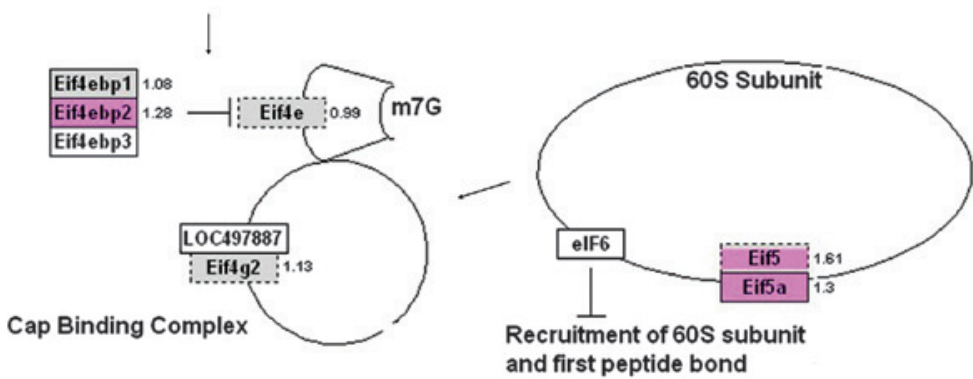

B

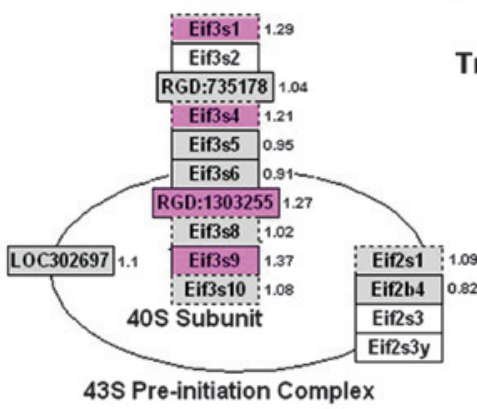

Translation factors

Elongation

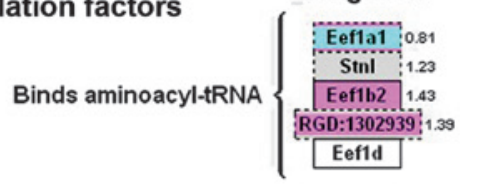

Termination

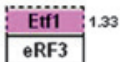

Translocation
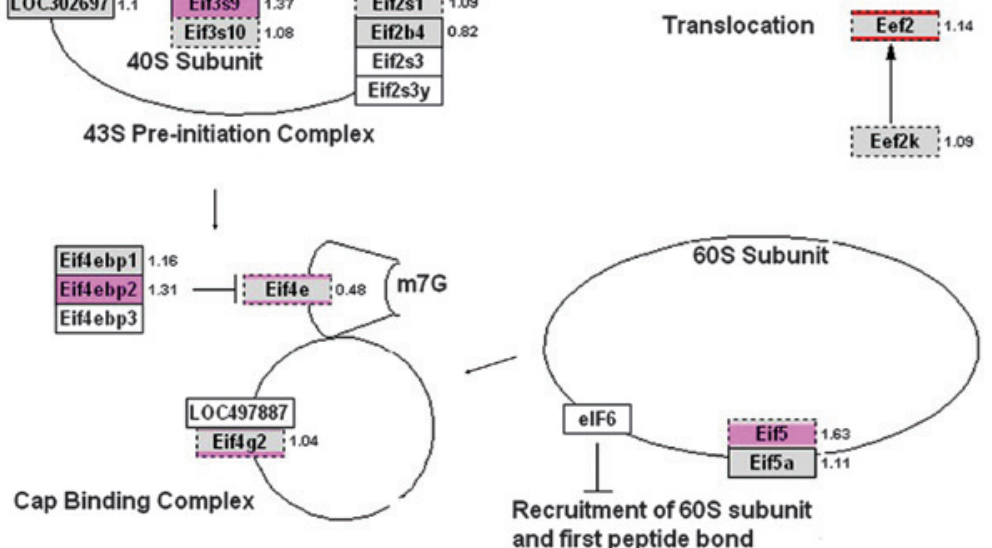

Figure 7. Analysis of signaling processes for factors involved in translation. Gene expression profiles of rat embryos prenatally exposed to (A) lipopolysaccharide (LPS) and (B) zymosan (Zym). The signaling process diagrams were overlaid with gene-expression colour criteria and ratios of gene-expression using the GenMAPP program. Red, upregulated (ratio >2); pink, upregulated (ratio >1.2); light blue, downregulated (ratio >1.2); grey, no significant change; white, gene not expressed on the GeneChip Array.

method was adopted to perform qPCR analysis for the target genes and a housekeeping gene ( $\beta$-actin) for each sample. The amplified products were subjected to melting curve analysis to verify their specificity. For each sample, the concentration of a target gene was divided by that of the housekeeping gene to estimate the relative content of the target gene in the sample.

\section{Results}

Microarray analysis. To determine the differentially expressed genes, signal ratios of $>2.0$ and $<0.5$ were used as the cut-off values for upregulated and downregulated genes, respectively. Rat embryos in the LPS group produced
183 upregulated and 270 downregulated genes, while rat embryos in the Zym group generated 144 upregulated and 417 downregulated genes, both compared with the control group. Results of our previous studies demonstrated that the prenatal administration of either LPS or Zym caused elevated blood pressure levels in rat offspring. Consequently, this study aimed to identify genes that changed in the same direction in both the LPS and Zym groups. A total of 50 upregulated and 173 downregulated genes occurred in both the LPS and Zym groups. These genes included a number of transcripts with unknown functions and several external sequence tags (ESTs). With regard to genes with a known function and that were shared by the two groups, 10 were upregulated and 85 were downregulated. 


\section{G-protein signaling and fibrotic response}

A
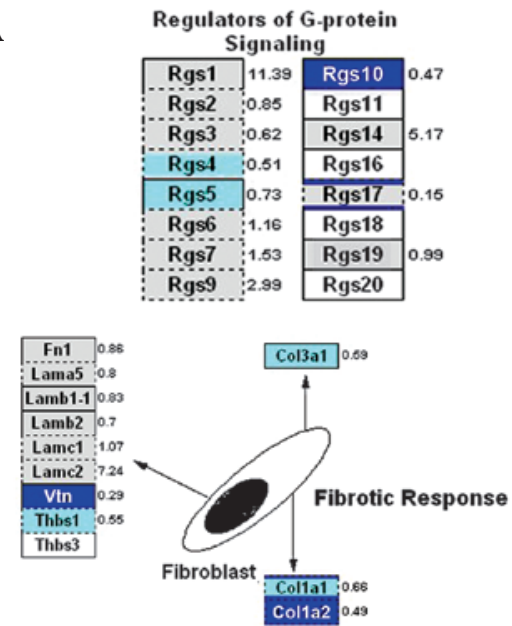

B
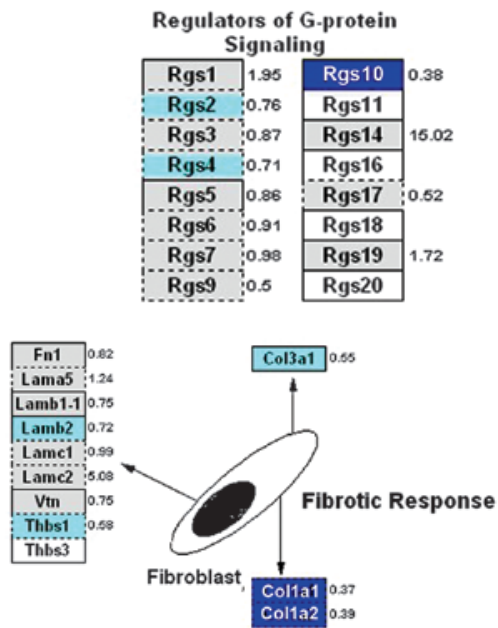

Figure 8. Analysis of genes involved in G-protein signaling and the fibrotic response. Gene expression profiles of rat embryos prenatally exposed to (A) lipopolysaccharide (LPS) and (B) zymosan (Zym). The signaling process diagrams were overlaid with gene-expression colour criteria and ratios of gene-expression using the GenMAPP program. Blue, downregulated (ratio $>2$ ); light blue, downregulated (ratio $>1.2$ ); grey, no significant change; white, gene not expressed on the GeneChip Array.

Biological function analysis. Functional analysis was classified based on biological function annotations in the GO database, and was mainly performed using three major characteristics (biological processes, molecular functions and cellular components). The biological process analysis showed that there were fewer upregulated than downregulated genes, and that there were even fewer upregulated and more downregulated genes in the Zym group. The molecular function analysis demonstrated that the differentially expressed genes were involved in a variety of biological pathways. The pathways with more differentially expressed genes included genes involved in cellular processes, development, metabolism and biological regulation (Fig. 1). The cellular component analysis indicated that the experimental groups had fewer upregulated than downregulated genes (Fig. 2). The focus of the present study was the analysis of molecular function, which, as previously stated, involved more downregulated than upregulated genes, and this pattern was more apparent in the Zym group. Additionally, detailed functional analysis showed that the differentially expressed genes consisted primarily of binding molecules, proteins with catalytic activity, transporters, signal transduction molecules and transcriptional regulators (Fig. 3) (18-20).

GenMAPP signaling analysis. The Gene Microarray Pathway Profile 2.1 (GenMAPP 2.1) software was used for signaling pathway analysis. The database (Biological Pathway Map) was composed primarily by the KEGG pathway database (http:// www.genome.jp/kegg), which is a database provided by a number of biologists and is maintained by a group of bioinformaticians (21-23).

The analysis of genes associated with DNA replication displayed numerous pink and red expression levels, indicating that the corresponding genes were highly expressed in the experimental groups. Additionally, there was high consistency between the LPS and Zym groups. The upregulated genes included Orc11, Cdk2, Mcm10, Gmnn, Rfc5, Rpa3 and PCNA (Fig. 4). Analysis of the G1/S regulatory pathways showed that there was a similar pattern during DNA synthesis following cell division. In particular, the upregulated genes included Atm, Mcm family genes and Cdk2. Furthermore, Myt1 was downregulated (Fig. 5). Genes involved in mRNA processing also exhibited an upregulated pattern that was consistent in both experimental groups. The upregulated genes, including Hnrpa1, Snrpa and Sf3b4, were mainly involved in mRNA splicing (Fig. 6). Several of the translation factors were also upregulated in the experimental groups; however, none of these genes exhibited a $>2$-fold increase in expression. Nevertheless, these results indicated that the upregulated genes were dominant in the entire process (Fig. 7).

Expression of the majority of downregulated genes was observed in calcium regulation pathways, including those involved in G-protein signaling, CaM kinase, PKC pathways and fibrotic response. Downregulated genes that occurred in both experimental groups included Rgs10, Rgs4, Col3a1, Colla1 and Colla2 (Fig. 8).

\section{Discussion}

GO analysis was initially performed in order to analyze the data in this study. GO is a collection of databases established by the Gene Ontology Consortium, which aims to generate a gene product classification standard to annotate gene and protein functions for various species. Currently, GO contains $>12$ databases (18). GO is primarily classified into three features; biological processes (e.g., orderly integration of molecular functions leading to increased biological activities), molecular functions (e.g., functions of individual gene products) and cellular components (e.g., mainly referring to cellular and subcellular structures, locations and macromolecular complexes) $(19,20)$.

In the present study, the distribution of differentially expressed genes, in terms of their biological processes, was found to vary greatly. Pathways with increased numbers of differentially expressed genes were mainly involved in cellular processes, development, metabolism and biological regula- 
tion. Following the preliminary analysis, classification of the differential expression data into one category was difficult; therefore, our initial GO analysis focused on molecular function. The categories for the differentially expressed genes were determined to be binding molecules, proteins with catalytic activity, transporters, signal transduction molecules and transcriptional modulators, which included cytokines and signal transduction proteins, transcription factors, enzymes directly involved in metabolism and troponin. The binding molecules mainly included genes with protein products that are responsible for nucleic acid and protein binding. The nucleic acid binding molecules were mainly regulators of transcription and translation, while the protein binding molecules were predominantly receptor binding proteins and signaling proteins. There were more downregulated than upregulated genes. This downregulation of embryonic gene expression may be important in the development of rat offspring. Although their function is unclear, these genes may play distinct roles during embryonic development.

The GenMAPP software was used to analyze the signaling pathways, and the visualization method was used to present information regarding the up- or downregulation of factors from certain pathways and their fold-change in gene expression. Using the GenMAPP software, the differentially expressed genes from biological processes of interest were analyzed $(21,22)$. Our analyses showed that in DNA synthesis, G1/S regulation, RNA processing and translational factors, the differentially expressed genes were mostly upregulated and were highly consistent between the LPS and Zym groups. This finding suggests that inflammatory stimulation is highly associated with the upregulation of genes involved in DNA replication, transcription and translation in rat embryos. Among the most important genes, Orc11, Cdk2, Atm, Mcm10, Gmnn, Rfc5, Rpa3 and PCNA are all involved in DNA replication; Hnrpa1, Snrpa and Sf3b4 in mRNA processing; and Eif3s9, Eif4ebp2, Eif5, Eef1b2 and Etf1 in translation. Differentially expressed genes were mainly downregulated in the inflammatory response pathways. For example, Rgs10, Rgs4, Col3a1, Colla1 and Col1a2 were downregulated in both experimental groups. It remains unclear whether the downregulation of inflammatory response pathways is associated with feedback inhibition resulting from maternal inflammatory responses.

Future studies investigating gene expression and gene and protein function will allow the underlying molecular mechanisms of offspring hypertension resulting from inflammatory stimulation during pregnancy to be further elucidated. However, it is currently possible to identify a number of specific inflammatory factors that are important in the model of inflammation-induced hypertension in rodent offspring.

\section{Acknowledgements}

This study was supported by the National Natural Science Foundation of China (nos. 81072630, 30973523 and 30900527).

\section{References}

1. McMillen IC and Robinson JS: Developmental origins of the metabolic syndrome: prediction, plasticity, and programming. Physiol Rev 85: 571-633, 2005.
2. Barker DJ: The developmental origins of chronic adult disease. Acta Paediatr Suppl 93: 26-33, 2004.

3. Gluckman PD, Lillycrop KA, Vickers MH, Pleasants AB Phillips ES, et al: Metabolic plasticity during mammalian development is directionally dependent on early nutritional status. Proc Natl Acad Sci USA 104: 12796-12800, 2007.

4. Johnson AD, Newton CC, Chasman DI, Ehret GB, Johnson T, et al; Cohorts for Heart and Aging Research in Genomic Epidemiology Consortium; Global BPgen Consortium; Women's Genome Health Study: Association of hypertension drug target genes with blood pressure and hypertension in 86,588 individuals. Hypertension 57: 903-910, 2011.

5. Lima SG, Hatagima A and Silva NL: Renin-angiotensin system: is it possible to identify hypertension susceptibility genes? Arq Bras Cardiol 89: 427-433, 2007.

6. Marcano AC, Onipinla AK, Caulfield MJ and Munroe PB: Recent advances in the identification of genes for human hypertension. Expert Rev Cardiovasc Ther 3: 733-741, 2005.

7. McBride MW, Graham D, Delles C and Dominiczak AF: Functional genomics in hypertension. Curr Opin Nephrol Hypertens 15: 145-151, 2006.

8. Bautista LE, Vera LM, Arenas IA and Gamarra G: Independent association between inflammatory markers $(\mathrm{C}$-reactive protein, interleukin-6, and TNF- $\alpha$ ) and essential hypertension. J Hum Hypertens 19: 149-154, 2005.

9. Sesso HD, Buring JE, Rifai N, Blake GJ, Gaziano JM and Ridker PM: C-reactive protein and the risk of developing hypertension. JAMA 290: 2945-2951, 2003.

10. Parissis JT, Korovesis S, Giazitzoglou E, Kalivas P and Katritsis D: Plasma profiles of peripheral monocyte-related inflammatory markers in patients with arterial hypertension. Correlations with plasma endothelin-1. Int J Cardiol 83: 13-21, 2002.

11. Samuelsson AM, Ohrn I, Dahlgren J, Eriksson E, Angelin B, et al: Prenatal exposure to interleukin-6 results in hypertension and increased hypothalamic-pituitary-adrenal axis activity in adult rats. Endocrinology 145: 4897-4911, 2004.

12. Samuelsson AM, Jennische E, Hansson HA and Holmäng A: Prenatal exposure to interleukin- 6 results in inflammatory neurodegeneration in hippocampus with NMDA/GABA(A) dysregulation and impaired spatial learning. Am J Physiol Regul Integr Comp Physiol 290: R1345-R1356, 2006.

13. Pauletto P and Rattazzi M: Inflammation and hypertension: the search for a link. Nephrol Dial Transplant 21: 850-853, 2006.

14. Wei YL, Li XH and Zhou JZ: Prenatal exposure to lipopolysaccharide results in increases in blood pressure and body weight in rats. Acta Pharmacol Sin 28: 651-656, 2007.

15. Liao W, Wei Y, Yu C, Zhou J, Li S, et al: Prenatal exposure to zymosan results in hypertension in adult offspring rats. Clin Exp Pharmacol Physiol 35: 1413-1418, 2008.

16. Hao XQ, Zhang HG, Yuan ZB, Yang DL, Hao LY, et al: Prenatal exposure to lipopolysaccharide alters the intrarenal renin-angiotensin system and renal damage in offspring rats. Hypertens Res 33: 76-82, 2010.

17. Hao XQ, Zhang HG, Li SH, Jia Y, Liu Y, et al: Prenatal exposure to inflammation induced by zymosan results in activation of intrarenal renin-angiotensin system in adult offspring rats. Inflammation 33: 408-414, 2010.

18. Ashburner M, Ball CA, Blake JA, Botstein D, Butler H, et al: Gene ontology: tool for the unification of biology. The Gene Ontology Consortium. Nat Genet 25: 25-29, 2000.

19. Zhou X and Su Z: EasyGO: Gene Ontology-based annotation and functional enrichment analysis tool for agronomical species. BMC Genomics 8: 246, 2007.

20. Alam-Faruque Y, Huntley RP, Khodiyar VK, Camon EB, Dimmer EC, et al: The impact of focused Gene Ontology curation of specific mammalian systems. PLoS One 6: e27541, 2011.

21. Dahlquist KD, Salomonis N, Vranizan K, Lawlor SC and Conklin BR: GenMAPP, a new tool for viewing and analyzing microarray data on biological pathways. Nat Genet 31: 19-20, 2002.

22. Salomonis N, Hanspers K, Zambon AC, Vranizan K, Lawlor SC, et al: GenMAPP 2: new features and resources for pathway analysis. BMC Bioinformatics 8: 217, 2007.

23. Doniger SW, Salomonis N, Dahlquist KD, Vranizan K, Lawlor SC and Conklin BR: MAPPFinder: using Gene Ontology and GenMAPP to create a global gene-expression profile from microarray data. Genome Biol 4: R7, 2003. 